\title{
Assessment of nutritional knowledge of soldiers of the Territorial Defense Force of the Polish Armed Forces
}

\section{Abstract}

Introduction: Among all types of the Armed Forces of the Republic of Poland, the name of the Territorial Defense Force reflects its character and purpose to the greatest extent. The goals and tasks set for it depend on the specific defense needs of the state. In the light of current nutritional knowledge, rational nutrition is one of the basic conditions for the proper functioning of the human body and maintaining its good health. Particularly important is the state of nutrition in the case of soldiers performing combat tasks. The aim of the study was to assess the level of nutritional knowledge of soldiers in the context of the implementation of training tasks.

Material and methods: The nutritional knowledge of soldiers was verified on the basis of an open survey, with the use of a proprietary questionnaire containing 20 closed-ended questions. The study covered 106 soldiers of the Territorial Defense Force, undergoing military training. The questions included in the questionnaire concerned the principles of rational nutrition and the sources of some of the nutrients in a daily diet. In addition, the Body Mass Index (BMI) was calculated for each respondent based on the height and body weight values. The results of the survey were prepared using Excel and Statistica software.

Results and discussion: The applied BMI classification showed this may be evidenced by over $40 \%$ of the respondents being overweight, while $17 \%$ demonstrated first degree obesity, and $1 \%$ - second degree obesity. The results of the survey indicated the areas in which the nutritional knowledge of the examined group of soldiers was insufficient. One of the reasons for this is the source of information on rational nutrition. Content provided by friends or obtained from the Internet may mislead soldiers, and incorrect application of nutrition guidelines may cause them to eat unbalanced food rations, which is likely to reduce a soldier's physical capacity, concentration, responsiveness, and the possibility of intense physical activity during the performance of official tasks. Taking into account the BMI results and the low level of nutritional knowledge, it can be concluded that the nutrition safety of the Territorial Defense Force soldiers is at risk, which may stimulate the emergence and development of a number of diet-related civilization diseases. It is purposeful to conduct systematic training of Territorial Defense Force soldiers in the field of rational nutrition. This will help to improve their nutritional knowledge and maintain good health.

\section{Conflict of Interest}

There is no conflict of interest 